\title{
Self-Consistent Sources and Conservation Laws for Super Coupled Burgers Equation Hierarchy
}

\author{
Si-Xing Tao
}

\begin{abstract}
Based upon the basis of Lie super algebra $B(0,1)$, the super coupled Burgers equation hierarchy with self-consistent sources was presented. Furthermore, the infinite conservation laws of above hierarchy were given.
\end{abstract}

Index Terms - Super coupled Burgers hierarchy, self-consistent sources, conservation laws, lie super algebras.

\section{INTRODUCTION}

Soliton equations with self-consistent sources have been receiving growing attention in recent years. Physically, the sources may result in solitary waves with a non-constant velocity and therefore lead to a variety of dynamics of physical models. For applications, these kinds of systems are usually used to describe interactions between different solitary waves and are relevant to some problems of hydrodynamics, solid state physics, plasma physics, etc. Ma, Strampp and Fuchssteiner systematically applied explicit symmetry constraint and binary nonlinearization of Lax pairs for generating the solution equation with sources [1], [2]. Furthermore, Ma presented the soliton solutions of the Schr ${ }^{0}$ dinger equation with self-consistent sources [3]. The discrete case of using variational derivatives in generating sources was discussed [4].

It is known that conservation laws play an important role on discussing the integrability for soliton equations. Since the discovery of infinite conservation laws for $\mathrm{KdV}$ equation by MGK [5], lots of methods have been developed to find them. This should be mainly due to the contribution of Wadati et al. [6]. Conservation laws also play an important part in mathematics as well.

With the development of soliton theory, super integrable systems associated with fermi variables have been receiving growing attention. Various methods have been developed to search for new super integrable systems, Lax pairs, soliton solutions, symmetries and conservation laws, et al. [7]-[18]. In 1997, Hu proposed the supertrace identity and applied it to establish the super Hamiltonian structures of super-integrable systems [7]. Then Professor Ma gave a systematic proof of super trace identity and presented the super Hamiltonian structures of super AKNS hierarchy and super Dirac hierarchy for application [8]. The super coupled Burgers hierarchy and its super-Hamiltonian structure were considered [9]. Recently, Yu et al considered the binary nonlinearization of the super AKNS hierarchy under an

Manuscript received March 3, 2013; revised May 17, 2013.

S. X. Tao is with School of Mathematics and Information Science, Shangqiu Normal University, Henan, Shangqiu 476000, China (e-mail: taosixing@163.com). implicit symmetry constraint [10] and the Bargmann symmetry constraint and binary nonlinearization of the super Dirac systems [11]. Meanwhile, various systematic methods on classical integrable systems have been developed to obtain exact solutions of the super integrable such as the inverse transformations, the $\mathrm{B}^{\ddot{\alpha}}$ cklund and Darboux transformations, the bilinear transformation of Hirota and others [19]-[21].

This paper is organized as follows. In section 2, the method for establishing super integrable soliton hierarchy with self-consistent sources by using Lie super algebra B $(0,1)$ was presented. For application, the super coupled Burgers hierarchy with self-consistent sources was obtained in Section III. In Section IV, the infinite conservation laws of the super coupled Burgers hierarchy were given.

\section{A KIND OF SUPER INTEGRABLE SOLITON HIERARCHY WITH SELF-CONSISTENT SOURCES}

In the following. Consider a basis of Lie super algebra $\mathrm{B}(0$, 1) $[8]$.

$$
\begin{aligned}
& e_{1}=\left(\begin{array}{ccc}
1 & 0 & 0 \\
0 & -1 & 0 \\
0 & 0 & 0
\end{array}\right), e_{2}=\left(\begin{array}{lll}
0 & 1 & 0 \\
1 & 0 & 0 \\
0 & 0 & 0
\end{array}\right), e_{3}=\left(\begin{array}{ccc}
0 & 1 & 0 \\
-1 & 0 & 0 \\
0 & 0 & 0
\end{array}\right), \\
& e_{4}=\left(\begin{array}{ccc}
0 & 0 & 1 \\
0 & 0 & 0 \\
0 & -1 & 0
\end{array}\right), e_{5}=\left(\begin{array}{lll}
0 & 0 & 0 \\
0 & 0 & 1 \\
1 & 0 & 0
\end{array}\right) .
\end{aligned}
$$

We introduce the loop algebra $\tilde{B}(0,1)$ as follows:

$$
\tilde{B}(0,1)=\{A \mid A \in R(\lambda) \otimes B(0,1)\} .
$$

where the loop algebra $\tilde{B}(0,1)$ is defined by span $\left\{\lambda^{n} \mid n \geq 0, A \in B(0,1)\right\}$.

Consider the auxiliary linear problem

$$
\begin{aligned}
& \left(\begin{array}{l}
\phi_{1} \\
\phi_{2} \\
\phi_{3}
\end{array}\right)_{x}=U(u, \lambda)\left(\begin{array}{l}
\phi_{1} \\
\phi_{2} \\
\phi_{3}
\end{array}\right), U(u, \lambda)=e_{0}(\lambda)+\sum_{i=1}^{5} u_{i} e_{i}(\lambda), \\
& \left(\begin{array}{l}
\phi_{1} \\
\phi_{2} \\
\phi_{3}
\end{array}\right)_{t_{n}}=V(u, \lambda)\left(\begin{array}{l}
\phi_{1} \\
\phi_{2} \\
\phi_{3}
\end{array}\right),
\end{aligned}
$$


where $u=\left(u_{1}, \cdots, u_{s}\right)^{T}, U(u, \lambda)=u_{1} e_{1}+\cdots+u_{p} e_{p}$,

$u_{i}=u_{i}(x, t)(i=1,2, \cdots, p), \phi_{i}=\phi_{i}(x, t)$ are field variables defining on $x \in R, t \in R, e_{i}=e_{i}(\lambda) \in \tilde{B}(0,1)$.

From the spectral problem (3), the compatibility condition gives rise to the well-known zero curvature equation

$$
U_{t_{n}}-V_{x}+[U, V]=0, n=1,2, \cdots,
$$

The general scheme of searching for the consistent $V^{(n)}$ and generating a hierarchy of nonlinear equations was proposed as follows [8]. We solve the equation

$$
V_{x}=[U, V], V=\sum_{m=0}^{\infty} V_{m} \lambda^{-m}=\sum_{m=0}^{\infty} \lambda^{-m}\left(\begin{array}{ccc}
A_{m} & B_{m}+C_{m} & \rho_{m} \\
B_{m}-C_{m} & -A_{m} & \delta_{m} \\
\delta_{m} & -\rho_{m} & 0
\end{array}\right),
$$

and search for $\Delta_{n}(u, \lambda) \in \tilde{B}(0,1)$, such that $V^{(n)}$ can be constructed by

$$
V^{(n)}=\sum_{m=0}^{n} V_{m} \lambda^{n-m}+\Delta_{n}(u, \lambda)
$$

and

$$
\Delta_{n}(u, \lambda)=\left(\begin{array}{ccc}
\Delta_{n 1} & \Delta_{n 2}+\Delta_{n 3} & \Delta_{n 4} \\
\Delta_{n 2}-\Delta_{n 3} & -\Delta_{n 1} & \Delta_{n 5} \\
\Delta_{n 5} & -\Delta_{n 4} & 0
\end{array}\right)
$$

where $\Delta_{n i}(1 \leq i \leq 5)$ are linear functions of $A_{m}, B_{m}, C_{m}$, $\rho_{m}, \delta_{m}$.

We consider the super trace identity of super integrable systems [8]

$$
\frac{\delta}{\delta u}\left(\operatorname{Str}\left(V \frac{\partial U}{\partial \lambda}\right)\right)=\lambda^{-\gamma} \frac{\partial}{\partial \lambda} \lambda^{\gamma} \operatorname{Str}\left(\frac{\partial U}{\partial u} V\right)
$$

Defining a scalar $H=H(u, \lambda)$ by the equation

$$
H=\operatorname{Str}\left(V \frac{\partial U}{\partial \lambda}\right), H=\sum_{m=0}^{\infty} H_{m}(u, \lambda) \lambda^{-m} .
$$

The sets $\left\{H_{m}\right\}$ prove the conserved densities of (4). The Hmailtonian form with $H_{n+1}$ can be written as

$$
\begin{gathered}
u_{t_{n}}=J \frac{\delta H_{n+1}}{\delta u}, n=1,2, \cdots \\
\frac{\delta H_{n}}{\delta u}=L \frac{\delta H_{n-1}}{\delta u}=\cdots=L^{n} \frac{\delta H_{0}}{\delta u}, n=1,2, \cdots
\end{gathered}
$$

where $L$ is a recursion operator and $J$ is a symplectic operator, and $\frac{\delta}{\delta u}=\left(\frac{\delta}{\delta u_{1}}, \cdots, \frac{\delta}{\delta u_{p}}\right)^{T}$.

According to (3) and (5), we consider the auxiliary linear problem. For $N$ distinct $\lambda_{j}, j=1, \cdots, N$, the following systems result from (1)

$$
\begin{gathered}
\left(\begin{array}{l}
\phi_{1 j} \\
\phi_{2 j} \\
\phi_{3 j}
\end{array}\right)_{x}=U\left(u, \lambda_{j}\right)\left(\begin{array}{l}
\phi_{1 j} \\
\phi_{2 j} \\
\phi_{3 j}
\end{array}\right)=\sum_{i=1}^{5} u_{i} e_{i}\left(\lambda_{j}\right)\left(\begin{array}{l}
\phi_{1 j} \\
\phi_{2 j} \\
\phi_{3 j}
\end{array}\right), \\
\left(\begin{array}{l}
\phi_{1 j} \\
\phi_{2 j} \\
\phi_{3 j}
\end{array}\right)_{t_{n}}=V^{(n)}\left(u, \lambda_{j}\right)\left(\begin{array}{l}
\phi_{1 j} \\
\phi_{2 j} \\
\phi_{3 j}
\end{array}\right) \\
=\left[\sum_{m=0}^{n} V_{m}\left(u, \lambda_{j}\right) \lambda_{j}^{n-m}+\Delta_{n}\left(u, \lambda_{j}\right)\right]\left(\begin{array}{l}
\phi_{1 j} \\
\phi_{2 j} \\
\phi_{3 j}
\end{array}\right) .
\end{gathered}
$$

Based on the results in [8], we show that the following equations

$$
\frac{\delta H_{k}}{\delta u}+\sum_{j=1}^{N} \alpha_{j} \frac{\delta \lambda_{j}}{\delta u}=0
$$

where $\alpha_{j}$ are constants. Equation (13) determines a finite dimensional invariant set for the flows (11).

For (12a), it is known that

$$
\frac{\delta \lambda_{j}}{\delta u}=\operatorname{Str}\left(\psi_{j} \frac{\partial U\left(u, \lambda_{j}\right)}{\partial u}\right)=\operatorname{Str}\left(\psi_{j} e_{i}\left(\lambda_{j}\right)\right), i=1, \cdots, 5 .
$$

where Str denotes the super trace of a matrix and

$$
\psi_{j}=\left(\begin{array}{ccc}
\phi_{1 j} \phi_{2 j} & -\phi_{1 j}^{2} & \phi_{1 j} \phi_{3 j} \\
\phi_{2 j}^{2} & -\phi_{1 j} \phi_{2 j} & \phi_{2 j} \phi_{3 j} \\
\phi_{2 j} \phi_{3 j} & -\phi_{1 j} \phi_{3 j} & 0
\end{array}\right), j=1, \cdots, N \text {. }
$$

According to (13), for a specific $k_{0} \geq n_{0}$, we demand that

$$
\frac{\delta H_{k_{0}}}{\delta u_{i}}=\sum_{j=1}^{N} \frac{\delta \lambda_{j}}{\delta u_{i}}=\sum_{j=1}^{N} \operatorname{Str}\left(\psi_{j} e_{i}\left(\lambda_{j}\right)\right)
$$

From (10) and (13), a kind of super integrable hierarchy with self-consistent sources can be present as follows

$$
u_{i, t_{n}}=J \frac{\delta H_{n+1}}{\delta u_{i}}+J \sum_{j=1}^{N} \frac{\delta \lambda_{j}}{\delta u_{i}}=J L^{n} \frac{\delta H_{1}}{\delta u_{i}}+J \sum_{j=1}^{N} \frac{\delta \lambda_{j}}{\delta u_{i}}, n=1,2, \cdots
$$




\section{THE SuPER COUPLED BURGERS HIERARCHY WITH SELF-CONSISTENT SOURCES}

The super Tu spectral problem associated with Lie super algebra $\mathrm{B}(0,1)$ is given by [9]

$$
\phi_{x}=U \phi, U=\left(\begin{array}{ccc}
q & r-\lambda+1 & \alpha \\
r-\lambda-1 & -q & \beta \\
\beta & -\alpha & 0
\end{array}\right), u=\left(\begin{array}{l}
q \\
r \\
\alpha \\
\beta
\end{array}\right), \phi=\left(\begin{array}{l}
\phi_{1} \\
\phi_{2} \\
\phi_{3}
\end{array}\right) \text {, }
$$

where $\lambda$ is a spectral parameter, $q$ and $r$ are even variables, $\alpha$ and $\beta$ are odd variables [9].

Taking

$$
V=\left(\begin{array}{ccc}
A & B+C & \rho \\
B-C & -A & \delta \\
\delta & -\rho & 0
\end{array}\right)
$$

the co-adjoint equation associated with (18) $V_{x}=[U, V]$ gives

$$
\left\{\begin{array}{c}
A_{x}=2 B-2 r C+2 \lambda C+\beta \rho+\alpha \delta, \\
B_{x}=2 q C-2 A-\alpha \rho+\beta \delta, \\
C_{x}=2 q B-2 r A+2 \lambda A-\alpha \rho-\beta \delta, \\
\rho_{x}=-\alpha A-\beta B-\beta C+q \rho-\lambda \delta+r \delta+\delta, \\
\delta_{x}=\beta A-\alpha B+\alpha C-\rho+r \rho-\lambda \rho-q \delta .
\end{array}\right.
$$

If we set

$$
A=\sum_{i \geq 0} A_{i} \lambda^{-i}, B=\sum_{i \geq 0} B_{i} \lambda^{-i}, C=\sum_{i \geq 0} C_{i} \lambda^{-i}, \rho=\sum_{i \geq 0} \rho_{i} \lambda^{-i}, \delta=\sum_{i \geq 0} \delta_{i} \lambda^{-i}
$$

then (19) is equivalent to

$$
\left\{\begin{array}{c}
A_{i+1}=r A_{i}-q B_{i}+\frac{1}{2} C_{i, x}+\frac{1}{2} \alpha \rho_{i}+\frac{1}{2} \beta \delta_{i}, \\
C_{i+1}=\frac{1}{2} A_{i, x}-B_{i}+r C_{i}-\frac{1}{2} \beta \rho_{i}-\frac{1}{2} \alpha \delta_{i}, \\
\rho_{i+1}=\beta A_{i}-\alpha B_{i}+\alpha C_{i}-\rho_{i}+r \rho_{i}-q \delta_{i}-\delta_{i, x}, \\
\delta_{i+1}=-\alpha A_{i}-\beta B_{i}-\beta C_{i}-\rho_{i, x}+q \rho_{i}+r \delta_{i}+\delta_{i}, \\
B_{i+1, x}=-2 A_{i+1}+2 q C_{i+1}-\alpha \rho_{i+1}+\beta \delta_{i+1}, i \geq 0 .
\end{array}\right.
$$

which results in the recurrence relations

$$
\left\{\begin{array}{c}
\left(A_{i+1}, B_{i+1}, \delta_{i+1},-\rho_{i+1}\right)^{T}=L\left(A_{i}, B_{i}, \delta_{i},-\rho_{i}\right)^{T}, \\
B_{i}=\partial^{-1}\left(-2 A_{i}+2 q C_{i}-\alpha \rho_{i}+\beta \delta_{i}\right), i \geq 0 .
\end{array}\right.
$$

where

$$
L=\left(\begin{array}{cccc}
\frac{1}{2} \partial \frac{1}{q} & \frac{1}{4} \partial q \partial-q & -\frac{1}{4} \partial \frac{\beta}{q}+\frac{1}{2} \beta & -\frac{1}{4} \partial \frac{\alpha}{q}-\frac{1}{2} \alpha \\
\partial^{-1} q \partial-\frac{1}{q} & \partial^{-1} r \partial-\frac{1}{2 q} \partial & \partial^{-1} \alpha \partial+\frac{\beta}{2 q} & \partial^{-1} \beta \partial+\frac{\alpha}{2 q} \\
-\alpha-\frac{\beta}{q} & -\beta-\frac{\beta}{2 q} \partial & r+1 & \partial-q-\frac{\alpha \beta}{2 q} \\
-\beta-\frac{\alpha}{q} & \alpha-\frac{\alpha}{2 q} \partial & \partial+q+\frac{\alpha \beta}{2 q} & r-1
\end{array}\right) .
$$

Upon choosing the initial conditions

$$
B_{0}=C_{0}=\rho_{0}=\delta_{0}=0, A_{0}=1,
$$

all other $A_{i}, B_{i}, C_{i}, \rho_{i}, \delta_{i}(i \geq 1)$ can be worked out by the recurrence relations (22). The first few sets are as follows:

$$
\begin{aligned}
& A_{1}=-q, B_{1}=0, C_{1}=-1, \rho_{1}=-\alpha, \delta_{1}=-\beta, A_{2}=-q r, \\
& B_{2}=-\frac{1}{2} q^{2}-\alpha \beta, C_{2}=-\frac{1}{2} q_{x}-r, \rho_{2}=\beta_{x}-r \alpha, \delta_{2}=\alpha_{x}-r \beta, \\
& A_{3}=-q r^{2}+\frac{1}{2} q^{3}+q \alpha \beta-\frac{1}{4} q_{x x}-\frac{1}{2} r_{x}+\frac{1}{2} \alpha \beta_{x}-\frac{1}{2} \alpha_{x} \beta, \\
& B_{3}=\frac{1}{2} q_{x}+r-q^{2} r+\alpha \alpha_{x}-\beta \beta_{x}-2 r \alpha \beta, \\
& C_{3}=-q_{x} r-\frac{1}{2} q r_{x}+\frac{1}{2} q^{2}+\alpha \beta-r^{2}-\frac{1}{2} \alpha \alpha_{x}-\frac{1}{2} \beta \beta_{x}, \\
& \rho_{3}=-\alpha_{x x}+\frac{1}{2} q^{2} \alpha-\frac{1}{2} q_{x} \alpha-\beta_{x}-r^{2} \alpha-q \alpha_{x}+r_{x} \beta+2 r \beta_{x}, \\
& \delta_{3}=-\beta_{x x}+\frac{1}{2} q^{2} \beta+\frac{1}{2} q_{x} \beta+r_{x} \alpha+2 r \alpha_{x}+q \beta_{x}-r^{2} \beta+\alpha_{x} .
\end{aligned}
$$

Let us associate the problem (18) with the following auxiliary problem

$$
\phi_{t_{n}}=V^{(n)} \phi
$$

with

$$
V^{(n)}=\sum_{i=0}^{n}\left(\begin{array}{ccc}
A_{i} & B_{i}+C_{i} & \rho_{i} \\
B_{i}-C_{i} & -A_{i} & \delta_{i} \\
\delta_{i} & -\rho_{i} & 0
\end{array}\right) \lambda^{n-i}+\left(\begin{array}{ccc}
0 & \frac{A_{n+1}}{q} & 0 \\
\frac{A_{n+1}}{q} & 0 & 0 \\
0 & 0 & 0
\end{array}\right) .
$$

The compatible conditions of the spectral problem (18) and the auxiliary problem (24) are

$$
U_{t_{n}}-V_{x}^{(n)}+\left[U, V^{(n)}\right]=0,
$$

which refer the super coupled Burgers soliton hierarchy

$$
\begin{aligned}
u_{t_{n}} & =K_{n} \\
& =\left(2 C_{n+1}-\frac{2 A_{n+1}}{q},\left(\frac{A_{n+1}}{q}\right)_{x},-\delta_{n+1}+\frac{\beta}{q} A_{n+1},-\rho_{n+1}+\frac{\alpha}{q} A_{n+1}\right)^{T} .
\end{aligned}
$$

Here $u_{t_{n}}=K_{n}$ in (26) is called the $n$-th coupled Burgers flow of this hierarchy.

Using the super trace identity

$$
\frac{\delta}{\delta u}\left(\operatorname{Str}\left(V \frac{\partial U}{\partial \lambda}\right)\right)=\lambda^{-\gamma} \frac{\partial}{\partial \lambda} \lambda^{\gamma} \operatorname{Str}\left(\frac{\partial U}{\partial u} V\right)
$$

where Str means the super trace[7,8], we have

$$
\left(\begin{array}{c}
A_{i+1} \\
B_{i+1} \\
\delta_{i+1} \\
-\rho_{i+1}
\end{array}\right)=\frac{\delta}{\delta u} H_{i}, H_{i}=\int \frac{B_{i+2}}{i+1} \mathrm{~d} x, i \geq 0 .
$$

Therefore, the super coupled Burgers soliton hierarchy (26) can be written as the following super Hamiltonian form:

$$
u_{t_{n}}=J \frac{\delta H_{n}}{\delta u},
$$


where

$$
J=\left(\begin{array}{cccc}
0 & \frac{1}{q} \partial & -\frac{1}{q} \beta & -\frac{1}{q} \alpha \\
\partial \frac{1}{q} & 0 & 0 & 0 \\
\frac{1}{q} \beta & 0 & -1 & 0 \\
\frac{1}{q} \alpha & 0 & 0 & 1
\end{array}\right)
$$

is a super symplectic operator, and $H_{n}$ is given by (28).

The first non-trivial nonlinear of super coupled Burgers hierarchy is given by its second flow

$$
\left\{\begin{array}{c}
q_{t_{2}}=\frac{1}{2 q} q_{x x}-2 q_{x} r-q r_{x}-\alpha \alpha_{x}-\beta \beta_{x}+\frac{r_{x}}{q}-\frac{\alpha \beta_{x}}{q}+\frac{\alpha_{x} \beta}{q}, \\
r_{t_{2}}=-2 r r_{x}+q q_{x}+\alpha_{x} \beta+\alpha \beta_{x}-\left(\frac{q_{x x}}{4 q}+\frac{r_{x}}{2 q}-\frac{\alpha \beta_{x}}{2 q}+\frac{\alpha_{x} \beta}{2 q}\right), \\
\alpha_{t_{2}}=\beta_{x x}-\frac{1}{2} q_{x} \beta-r_{x} \alpha-2 r \alpha_{x}-q \beta_{x}-\alpha_{x}-\frac{q_{x x} \beta}{2 q}-\frac{r_{x} \beta}{2 q}-\frac{\alpha \beta \beta_{x}}{2 q}, \\
\beta_{t_{2}}=\alpha_{x x}+\frac{1}{2} q_{x} \alpha+\beta_{x}+q \alpha_{x}-r_{x} \beta-2 r \beta_{x}-\frac{q_{x x} \alpha}{2 q}-\frac{r_{x} \alpha}{2 q}-\frac{\alpha \alpha_{x} \beta}{2 q} .
\end{array}\right.
$$

which possesses a Lax pair of $U$ defined in (18) and $V^{(2)}$ defined by

$$
V^{(2)}=\left(\begin{array}{ccc}
-q \lambda-q r & \lambda^{2}-\lambda-\frac{1}{2} q_{x}-r-\frac{1}{2} q^{2}-\alpha \beta & -\alpha \lambda+\beta_{x}-r \alpha \\
\lambda^{2}+\lambda+\frac{1}{2} q_{x}+r-\frac{1}{2} q^{2}-\alpha \beta & q \lambda+q r & -\beta \lambda+\alpha_{x}-r \beta \\
-\beta \lambda+\alpha_{x}-r \beta & \alpha \lambda-\beta_{x}+r \alpha & 0
\end{array}\right) .
$$

Next we will establish the super coupled Burgers hierarchy with self-consistent sources. Consider the linear system

$$
\begin{aligned}
& \left(\begin{array}{l}
\phi_{1 j} \\
\phi_{2 j} \\
\phi_{3 j}
\end{array}\right)_{x}=U\left(\begin{array}{l}
\phi_{1 j} \\
\phi_{2 j} \\
\phi_{3 j}
\end{array}\right)=\left(\begin{array}{ccc}
q & r-\lambda+1 & \alpha \\
r-\lambda-1 & -q & \beta \\
\beta & -\alpha & 0
\end{array}\right)\left(\begin{array}{l}
\phi_{1 j} \\
\phi_{2 j} \\
\phi_{3 j}
\end{array}\right), \\
& \left(\begin{array}{l}
\phi_{1 j} \\
\phi_{2 j} \\
\phi_{3 j}
\end{array}\right)_{t_{n}}=V\left(\begin{array}{l}
\phi_{1 j} \\
\phi_{2 j} \\
\phi_{3 j}
\end{array}\right)=\left(\begin{array}{ccc}
A & B+C & \rho \\
B-C & -A & \delta \\
\delta & -\rho & 0
\end{array}\right)\left(\begin{array}{l}
\phi_{1 j} \\
\phi_{2 j} \\
\phi_{3 j}
\end{array}\right) .
\end{aligned}
$$

For the system (31), we consider the $\frac{\delta H}{\delta u}=\sum_{j=1}^{N} \frac{\delta \lambda_{j}}{\delta u}$ in the Lie super algebra $B(0,1)$ and obtain

$$
\frac{\delta \lambda_{j}}{\delta u}=\left(\begin{array}{c}
\operatorname{Str}\left(\Psi_{j} \frac{\partial U}{\partial q}\right) \\
\operatorname{Str}\left(\Psi_{j} \frac{\partial U}{\partial r}\right) \\
\operatorname{Str}\left(\Psi_{j} \frac{\partial U}{\partial \alpha}\right) \\
\operatorname{Str}\left(\Psi_{j} \frac{\partial U}{\partial \beta}\right)
\end{array}\right)=\left(\begin{array}{c}
2\left\langle\Phi_{1}, \Phi_{2}\right\rangle \\
\left\langle\Phi_{2}, \Phi_{2}\right\rangle-\left\langle\Phi_{1}, \Phi_{1}\right\rangle \\
-2\left\langle\Phi_{2}, \Phi_{3}\right\rangle \\
2\left\langle\Phi_{1}, \Phi_{3}\right\rangle
\end{array}\right)
$$

where $\Phi_{i}=\left(\phi_{i 1}, \cdots, \phi_{i N}\right)^{T}(i=1,2,3)$.

According to the results in (17), the super coupled Burgers hierarchy with self-consistent sources is present

$$
u_{t_{n}}=\left(\begin{array}{c}
q \\
r \\
\alpha \\
\beta
\end{array}\right)_{t_{n}}=J L^{n}\left(\begin{array}{c}
-q \\
0 \\
-\beta \\
\beta
\end{array}\right)+J\left(\begin{array}{c}
2\left\langle\Phi_{1}, \Phi_{2}\right\rangle \\
\left\langle\Phi_{2}, \Phi_{2}\right\rangle-\left\langle\Phi_{1}, \Phi_{1}\right\rangle \\
-2\left\langle\Phi_{2}, \Phi_{3}\right\rangle \\
2\left\langle\Phi_{1}, \Phi_{3}\right\rangle
\end{array}\right) .
$$

The first nontrivial integrable super coupled Burgers hierarchy with self-consistent sources is its second flow

$$
\left\{\begin{array}{rl}
q_{t_{2}}= & \frac{1}{2 q} q_{x x}-2 q_{x} r-q r_{x}-\alpha \alpha_{x}-\beta \beta_{x}+\frac{r_{x}}{q}-\frac{\alpha \beta_{x}}{q}+\frac{\alpha_{x} \beta}{q} \\
& \frac{1}{q}\left(\left\langle\Phi_{2}, \Phi_{2}\right\rangle-\left\langle\Phi_{1}, \Phi_{1}\right\rangle\right)_{x}+\frac{2 \beta}{q}\left\langle\Phi_{2}, \Phi_{3}\right\rangle-\frac{2 \alpha}{q}\left\langle\Phi_{1}, \Phi_{3}\right\rangle, \\
r_{t_{2}}=-2 & r r_{x}+q q_{x}+\alpha_{x} \beta+\alpha \beta_{x}-\left(\frac{q_{x x}}{4 q}+\frac{r_{x}}{2 q}-\frac{\alpha \beta_{x}}{2 q}+\frac{\alpha_{x} \beta}{2 q}\right)_{x}+2\left(\frac{\left\langle\Phi_{1}, \Phi_{3}\right\rangle}{q}\right)_{x}, \\
\alpha_{t_{2}}= & \beta_{x x}-\frac{1}{2} q_{x} \beta-r_{x} \alpha-2 r \alpha_{x}-q \beta_{x}-\alpha_{x}-\frac{q_{x} \beta}{2 q}-\frac{r_{x} \beta}{2 q}-\frac{\alpha \beta \beta_{x}}{2 q} \\
& +\frac{2 \beta}{q}\left\langle\Phi_{1}, \Phi_{2}\right\rangle-2\left\langle\Phi_{2}, \Phi_{3}\right\rangle, \\
\beta_{t_{2}}= & \alpha_{x x}+\frac{1}{2} q_{x} \alpha+\beta_{x}+q \alpha_{x}-r_{x} \beta-2 r \beta_{x}-\frac{q_{x} \alpha}{2 q}-\frac{r_{x} \alpha}{2 q}-\frac{\alpha \alpha_{x} \beta}{2 q} \\
& +\frac{2 \alpha}{q}\left\langle\Phi_{1}, \Phi_{2}\right\rangle+2\left\langle\Phi_{1}, \Phi_{3}\right\rangle .
\end{array}\right.
$$

when $\alpha=\beta=0$, it is the well known nonlinear coupled Burgers equation with self-consistent sources. So system (33) is a novel super integrable equation hierarchy.

\section{CONSERVATION LAWS FOR THE SuPER COUPLED BURGERS HIERARCHY}

In what follows, we will construct conservation laws of the super coupled Burgers equation. Introduce the variables:

$$
K=\frac{\phi_{2}}{\phi_{1}}, G=\frac{\phi_{3}}{\phi_{1}},
$$

where $p(K)=0, p(G)=1$. From (12), we have

$$
\left\{\begin{array}{c}
K_{x}=r-\lambda-1-2 q K+\beta G-(r-\lambda+1) K^{2}-\alpha K G, \\
G_{x}=\beta-\alpha K-q G-(r-\lambda+1) K G-\alpha G^{2} .
\end{array}\right.
$$

We expand $K, G$ in powers of $\lambda^{-1}$ as follows

$$
K=\sum_{j=0}^{\infty} k_{j} \lambda^{-j}, G=\sum_{j=0}^{\infty} g_{j} \lambda^{-j}
$$

where $p\left(k_{j}\right)=0, p\left(g_{j}\right)=1$. Substituting (37) into (36) and comparing the coefficients of the same powers of $\lambda$, we obtain

$$
\begin{aligned}
& k_{0}=1, k_{1}=q+1, k_{2}=\frac{1}{2} q_{x}+(q+r+1)(q+1), \\
& k_{3}=\frac{3}{2} q q_{x}+\frac{1}{2} q r_{x}+\frac{1}{2} q_{x} r+q_{x}+\frac{1}{2} r_{x}+\frac{1}{4} q_{x x}+\frac{3}{2} q^{3} \\
& +\frac{3}{2} q^{2}+\frac{3}{2} q+1+\frac{1}{2} q^{2} r+\frac{3}{2} q r+r+\frac{1}{2} \alpha_{x} \beta+\frac{1}{2} \alpha \alpha_{x} \\
& +\frac{1}{2} \beta \beta_{x}-\frac{1}{2} \alpha \beta_{x}, g_{0}=0, g_{1}=\alpha-\beta, g_{2}=\alpha_{x}-\beta_{x} \\
& +2 q \alpha+2 \alpha+r \alpha-q \beta-r \beta-\beta, g_{3}=\alpha_{x x}-\beta_{x x}+ \\
& \frac{5}{2} q_{x} \alpha+3 q \alpha_{x}+2 \alpha_{x}+r_{x} \alpha+r \alpha_{x}-r_{x} \beta-r \beta_{x}-q_{x} \beta \\
& -2 q \beta_{x}-\beta_{x}+3 q^{2} \alpha+5 q \alpha+3 q r \alpha-2 q r \beta-q^{2} \beta \\
& -2 q \beta+2 r \alpha-r \beta+2 \alpha-\beta .
\end{aligned}
$$

and a recursion formula for $k_{n}$ and $g_{n}$,

$$
\left\{\begin{array}{l}
k_{n+1}=\frac{1}{2} k_{n x}+q k_{n}-\frac{1}{2} \beta g_{n}+\frac{1}{2}(r+1)\left(\sum_{l=0}^{n} k_{l} k_{n-l}\right)+\frac{1}{2} \alpha\left(\sum_{l=0}^{n} k_{l} g_{n-l}\right), \\
g_{n+1}=g_{n x}+\alpha k_{n}+q g_{n}+(r+1)\left(\sum_{l=0}^{n} k_{l} g_{n-l}\right)+\alpha\left(\sum_{l=0}^{n} g_{l} g_{n-l}\right), n \geq 3 .
\end{array}\right.
$$


Because of

$$
\frac{\partial}{\partial t} \frac{\phi_{1, x}}{\phi_{1}}=\frac{\partial}{\partial x} \frac{\phi_{1, t}}{\phi_{1}}
$$

we derive the conservation laws of (30)

$$
\frac{\partial}{\partial t}(q+(r-\lambda+1) K+\alpha G)=\frac{\partial}{\partial x}(A+(B-C) K+G)
$$

where

$$
\begin{aligned}
& A=-c_{0} \lambda-c_{0} q r-c_{1} q, B=c_{0} \lambda^{2}+c_{1} \lambda-\frac{1}{2} c_{0} q^{2}-c_{0} \alpha \beta, \\
& C=-c_{0} \lambda-\frac{1}{2} c_{0} q_{x}-c_{0} r-c_{1}, \rho=-c_{0} \alpha \lambda+c_{0} \beta_{x}-c_{0} r \alpha-c_{1} \alpha .
\end{aligned}
$$

\section{Assume}

that

$\sigma=q+(r-\lambda+1) K, \theta=A+(B+C) K+\rho G$, then (41) can be written as $\sigma_{t}=\theta_{x}$, which is the right form of conservation laws. We expand $\sigma$ and $\theta$ as series in powers of $\lambda$ according with the coefficients, which are called conserved densities and currents respectively

$$
\sigma=\sum_{j=0}^{\infty} \sigma_{j} \lambda^{-j}, \theta=c_{0} \lambda^{2}+c_{1} \lambda+\sum_{j=0}^{\infty} \theta_{j} \lambda^{-j},
$$

where $c_{0}, c_{1}$ are constants of integration. Then the first two conserved densities and currents are

$$
\begin{aligned}
\sigma_{0}= & r-q, \sigma_{1}=-\frac{1}{2} q_{x}-q^{2}-q-\alpha \beta, \\
\theta_{0}= & c_{0}\left(\frac{1}{2} q^{2}+q+q r\right)+c_{1} q, \\
\theta_{1}= & c_{0}\left(q q_{x}+\frac{1}{2} q r_{x}+\frac{1}{2} q_{x} r+\frac{1}{2} r_{x}+\frac{1}{4} q_{x x}\right. \\
& +q^{3}-\frac{1}{2} q+\frac{1}{2} q^{2} r-\frac{1}{2} q r-r+\frac{1}{2} \alpha_{x} \beta \\
& \left.-\frac{1}{2} \alpha \alpha_{x}-\frac{1}{2} \beta \beta_{x}+\frac{3}{2} \alpha \beta_{x}+2 r \alpha \beta\right) \\
& +c_{1}\left(\frac{1}{2} q_{x}+q^{2}+q r+q+r-\alpha \beta\right) .
\end{aligned}
$$

The recursion relations for $\sigma_{n}$ and $\theta_{n}$ are

$$
\left\{\begin{array}{c}
\sigma_{n}=(r+1) k_{n}-k_{n+1}+\alpha g_{n}, \\
\theta_{n}=c_{0}\left(k_{n+2}-\frac{1}{2} q^{2} k_{n}-\alpha \beta k_{n}-k_{n+1}-\frac{1}{2} q_{x} k_{n}-r k_{n}\right. \\
\left.-\alpha g_{n+1}+\beta_{x} g_{n}-r \alpha g_{n}\right)+c_{1}\left(k_{n+1}-k_{n}-\alpha g_{n}\right) .
\end{array}\right.
$$

where $k_{n}$ and $g_{n}$ can be calculated from (39). The infinitely conservations laws of (39) can be easily obtained in (35)-(43) respectively.

\section{ACKNOWLEDGMENT}

This work was supported by the National Natural Science Foundation of China under Grant No. 61072147, the Natural Science Foundation of Henan Province under Grant No.132300410202, the Science and Technology Key Research Foundation of the Education Department of Henan Province under Grant No.12A110017 and the Youth Research Foundation of Shangqiu Normal University under Grant No. 2011QN12.

\section{REFERENCES}

[1] W. X. Ma and W. Strampp, "An explicit symmetry constraint for the Lax pairs of AKNS systems," Phys. Lett. A, vol. 185, no. 3, pp. 277-286, Feb. 1994.

[2] W. X. Ma and B. Fuchssteiner, "Binary nonlinearization of Lax pairs," in Proc. the First Workshop on Nonlinear Physics, Italy, 1996, pp. 217-224.

[3] W. X. Ma, "Soliton, positon and negaton solutions to a schrdinger self-consistent source equation," J. Phys. Soc. Jpn., vol. 72, no. 12, pp. 3017-3019, Dec. 2003.

[4] W. X. Ma and X. G. Geng. "Bäcklund transformations of soliton systems from symmetry constraints," CRM Proc. Lect. Notes, vol. 29, 2001, pp. 313-323.

[5] R. M. Miura, C. S. Gardner, and M. D. Kruskal, "Korteweg-de vries equation and generalizations. ii. existence of conservation laws and constants of motion," J. Math. Phys., vol. 9, no. 8, pp. 1204-1209, Aug. 1968.

[6] M. Wadati, H. Sanuki, and K. Konno, "relationships among inverse method, bäcklund transformation and an infinite number of conservation laws," Prog. Theor. Phys., vol. 53, no. 2, pp. 419-436, Feb. 1975

[7] X. B. Hu, "An approach to generate superextensions of integrable systems," J. Phys. A: Math. Gen., vol. 30, no. 2, pp. 619-632, Jan. 1997.

[8] W. X. Ma, J. S. He, and Z. Y. Qin, "A supertrace identity and its applications to super integrable systems," J. Math. Phys., vol. 49, no. 3, pp. 033511, Mar. 2008.

[9] H. Shi and S. X. Tao, "Super coupled Burgers hierarchy and its super Hamiltonian structures," J. Zhengzhou Univ. (Nat. Sci. Ed.), vol. 44, no. 3, pp. 16-19, Sep. 2012.

[10] J. Yu, J. W. Han, and J. S. He, "Binary nonlinearization of the super AKNS system under an implicit symmetry constraint," J. Phys. A: Math. Theo., vol. 42, pp. 465201, Nov. 2009.

[11] J. Yu, J. S. He, W. X. Ma, and Y. Cheng, "The bargmann symmetry constraint and binary nonlinearization of the super dirac system," Chin. Ann. Math., vol. 31B, no. 3, pp. 361-372, May 2010.

[12] B. A. Kupershmidt, "A super korteweg-de vries equation: An integrable system," Phys. Lett. A, vol. 102, no. 5-6, pp. 213-215, May 1984.

[13] P. Mathieu, "Supersymmetric extension of the korteweg-de vries equation," J. Math. Phys., vol. 29, no. 11, pp. 2499-2507, Nov.1988.

[14] B. A. Kupershmidt, "Bosons and fermions interacting integrably with the korteweg-de vries field," J. Phys. A: Math. Gen., vol. 17, no. 16, pp. L869-L872, Nov. 1984.

[15] Y. S. Li and L. N. Zhang, "A note on the super AKNS equations," J. Phys. A: Math. Gen., vol. 21, no. 7, pp. 1549-1552, Apr. 1988.

[16] Y. I. Manin and A. O. Radul, "A supersymmetric extension of the kadomtsev-petviashvili hierarchy," Commun. Math. Phys., vol. 98, no. 1, pp. 65-77, Mar. 1985.

[17] J. C. Beuneli, A. Das, and Z. Popowicz, "Supersymmetric extensions of the Harry Dym hierarchy," J. Math. Phys., vol. 44, no. 10, pp. 4756-4767, Oct. 2003.

[18] C. Devchand and J. Schiff, "The supersymmetric Camassa-Holm equation and geodesic flow on the superconformal group," J. Math. Phys., vol. 42, no. 1, pp. 260-273, Jan. 2001.

[19] Q. P. Liu, "Darboux transformations for supersymmetric korteweg-de equations," Lett. Math. Phys., vol. 35, no. 2, pp. 115-122, Oct.1995.

[20] M. Siddiq, M. Hassan, and U. Saleem, "On darboux transformation of the supersymmetric sine-gordon equation," J. Phys. A: Math. Gen., vol. 39, no. 23, pp. 7313- 7318, Jun. 2006.

[21] Y. B. Zeng, W. X. Ma, and R. L. Lin, "Integration of the soliton hierarchy with self-consistent sources," J. Math. Phys., vol. 41, no. 8, pp. 5453-5489, Aug. 2000.

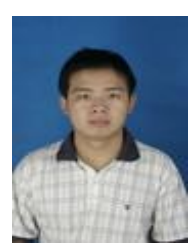

Si-Xing Tao was born in March 1981, in Qingzhou, Shandong Province of China. He received the bachelor degree in mathematics and applied mathematics from Qufu Normal University in 2003 and the master degree in Basic Mathematics from Qufu Normal University in 2006. He received the doctor degree in Basic Mathematics from Shanghai University in 2011. Now he is a lecturer in School of Mathematics and Information Science, Shangqiu Normal University. His research interests are in soliton theory and integrable systems and published more than 10 articles in recent years. 